\title{
REVIEW
}

\section{The SHP-2 tyrosine phosphatase: Signaling mechanisms and biological functions}

\author{
Qu Cheng Kui \\ Department of Hematopoiesis, Jerome H. Holland Laboratory \\ for the Biomedical Sciences, American Red Cross, Rockville, \\ Department of Anatomy and Cell Biology, George Washington \\ University School of Medicine and Health Sciences, Washing- \\ ton $D C$
}

\begin{abstract}
Cellular biological activities are tightly controlled by intracellular signaling processes initiated by extracellular signals. Protein tyrosine phosphatases, which remove phosphate groups from tyrosine phosphorylated signaling molecules, play equally important tyrosine roles as protein kinases in signal transduction. SHP-2, a cytoplasmic SH2 domain containing protein tyrosine phosphatase, is involved in the signaling pathways of a variety of growth factors and cytokines. Recent studies have clearly demonstrated that this phosphatase plays an important role in transducing signal relay from the cell surface to the nucleus, and is a critical intracellular regulator in mediating cell proliferation and differentiation.
\end{abstract}

Key words: SHP-2, SHP-1, Signal transduction.

\section{INTRODUCTION}

Cells can not survive without environmental factors. Cellular responses to a variety of extracellular cues are mediated by intracellular signaling pathways. A great deal of evidence has demonstrated that dysregulation of such signaling pathways initiated from extracellular factors causes malfunctioning of the targeted cells, and eventually

\footnotetext{
* Corresponding address: Dr. Cheng Kui Qu, Department of Hematopoiesis, Holland Laboratory, American Red Cross, 15601 Crabbs Branch Way, Rockville, MD 20855 USA

Tel: (301) 738-0445; Fax: (301) 738-0444; E-mail: quc@usa.redcross.org
} 
SHP-2 in Signal transduction

leads to diseases. Many important cellular activities, such as cell proliferation, differentiation, and death, are highly controlled by the cellular signal transduction processes in which protein phosphorylation and dephosphorylation are central events[1-3]. It is increasingly clear that these biochemical processes are carried out by protein tyrosine kinases (PTKs) and protein tyrosine phosphatases (PTPs). Protein phosphorylation and dephosphorylation are closely related to the activities of the signaling proteins and directly mediate protein-protein interaction. Thus, PTPs, which dephosphorylate tyrosine phosphorylated signaling molecules, play an equally important role as PTKs in transducing signal flow and controlling cellular behavior. However, studies regarding PTPs are lagging behind. SHP-2, a Src homology 2 (SH2) domain containing nontransmembrane PTP, has been demonstrated to be involved in a variety of cytokine and growth factor initiated signal transduction processes[4-8]. Increasing evidence has indicated that this phosphatase plays an important role in diverse signaling pathways to regulate cellular biological processes. Even though significant progress has been made in the last several years, many aspects of the activities of this phosphatase still remain unaddressed. This review will focus on the signaling mechanisms and the biological functions of this enzyme. Particular attention will be paid to its role in hematopoietic cell regulation.

\section{Signaling mechanisms}

SHP-2, previously called SH-PTP2, PTP1D, SH-PTP3, and Syp, was identified independently by several groups as a cytosolic $\mathrm{SH} 2$ domain containing protein tyrosine phosphatase[4-8]. It is ubiquitously expressed in various tissues and cell types. It has a similar overall structure and high homology with the hematopoietic cell specific-SHP-1 phosphatase[9],[10]. Both of these phosphatases contain two tandem SH2 domains at the $\mathrm{N}$-terminus and one phosphatase domain at the C-terminus. The SH2 domain is a 100 amino acid motif which mediates the binding of SHP-2 and SHP-1 to the phosphorylated tyrosine residues on other molecules, thus directing the specific protein-protein interaction for these two phosphatases[11]. Normally, the auto-inhibitory influence of their SH2 domains renders these two enzymes catalytically inefficient. Occupancy of the SH2 domains by phosphotyrosine residues leads to an increase in the catalytic activity of the SHP-2 and SHP-1 phosphatases, possibly by inducing conformational changes in the enzymes[12,13]. Accumulated biochemical data have shown that both SHP-2 and SHP-1 act downstream of receptor and cytoplasmic tyrosine kinases to propagate the signal relay from the cell surface to the nucleus. Despit high homology between SHP-2 and SHP-1, their functions might be distinct. Genetic analyses on Xenopus revealed that both the $\mathrm{SH} 2$ domains and the phosphatase domains contribute to signaling, and thus functional specificity of these two PTPs[14,15].

SHP-2 is expressed in various tissues and cell types, and has been implicated in diverse signaling pathways including those initiated by growth factors such as PDGF, 
EGF, and IGF-1, cytokines such as IL-3, GM-CSF, and EPO, as well as insulin and interferon[16,17]. SHP-2 has compound signaling functions. It appears to be involved in a variety of signal transduction processes, such as the Ras-Raf-MAP kinase, JakStat, and PI3 kinase pathways. Within a single signaling pathway, SHP-2 may act at multiple sites to participate in the signal relay. For instance, SHP-2 directly interacts with cytokine and growth factor receptors, such as PDGF, EGF, SCF, and EPO receptors. It has also been demonstrated to bind with a variety of signaling intermediates such as Grb2, FRS-2, Jak2, p85 subunit of PI3 kinase, IRS-1, and Gab1 and 2. As a protein tyrosine phosphatase, SHP-2 is believed to function by dephosphorylating its associated signaling molecules, thus diminishing the local signaling flow. However, the ultimate effect of SHP-2 action in most signaling pathways is to enhance the signal transduction. The precise mechanism remains to be defined.

In most circumstances, SHP-2 plays a positive role in transducing the signal relay from receptor PTKs[14], [18-21]. Previous biochemical evidence has demonstrated that the SHP-2 enzymatic activity is required for its function in signal transduction[21], [22]. Cysteine (Cys) at amino acid residue 459 has been identified as critical for its phosphatase activity. While the replacement of cysteine with serine (Ser) at this site completely abolishes its enzymatic activity, the capacity for this mutant molecule to bind to other signaling intermediates via its $\mathrm{SH} 2$ domains remains unaltered. This mutant functions thus as a dominant negative molecule over the endogenous wild type SHP-2. Using the dominant negative approach, SHP-2 has been reported, in a number of studies, to positively regulate the signaling pathways of insulin, EGF, PDGF, and FGF. Overexpression of this mutant protein has been shown to block the signaling functions of endogenous SHP-2 in both in vitro and in vivo models. For example, the introduction of a catalytically inert SHP-2 molecule markedly inhibited the activation of MAP kinase in response to EGF, insulin, and fibronectin stimulation[19], [21],[2326]. The precise biochemical basis for such positive regulation for this phosphatase still remains unclear. Biochemical studies on the Drosophila homologue of SHP-2, csw, revealed that SHP-2 may function at a parallel level or upstream of Ras in the MAP kinase pathway[27].

In some cases, SHP-2 does play a negative role in intracellular signaling processes, thus it may have dual functions in cytokine and growth factor signal transduction. For instance, SHP-2 negatively regulates the Jak-Stat signaling pathway initiated by interferon $-\alpha$ and $-\gamma$. As a consequence, SHP-2 mutant cells are more sensitive to the cytotoxicity of interferons, and the activation of downstream Stat2 and Stat1 is elevated[28]. Another prominent example is that SHP-2 was found to function to diminish the signal relay from gp130[29-31]. For instance, the recent knockin mouse model, in which the endogenous gp130 has been replaced with a human gp130 Y759F (equivalent to residue 757 in mouse) mutant, displayed lymphoadenopathy, splenomegaly, and an enhanced acute-phase response. However, the data supporting the negative regulatory role for SHP-2 is based on observed increases in gp130-dependent signaling when 
SHP-2 in Signal transduction

Y757 on gp130, a pivotal binding site for SHP-2, is mutated to phenylalanine. The question remaining to be resolved is whether these phenotypes are really mediated by SHP-2, because a more recent finding demonstrated that the Y757 is also the recognition site for SOCS-3, a putative SH2 domain containing cytokine signaling suppressor [32]. SOCS-3 and SHP-2 may compete to bind to this site, and SHP-2 functions to block the effects of SOCS-3 in gp 130 pathway.

SHP-2 is also highly expressed in hematopoietic cells, and has been indicated to be involved in hematopoietic growth factor signal transduction[33-35]. It has been shown to participate in the signal transduction from IL-3, EPO, SCF, GM-CSF, and IL-5. However, compared to SHP-1 phosphatase, understanding of the physiological and biochemical functions of SHP-2 in hematopoietic cells lags far behind. A potential role of SHP-2 phosphatase in hematopoietic cell signaling is indicated by indirect evidence based on receptor-mediated changes in SHP-2 tyrosine phosphorylation and/or its association with receptors or other signaling intermediates. Unfortunately, the cellular significance of SHP-2 involvement in these pathways remains to be elucidated. This could be achieved through the signal transduction studies on the hematopoietic cell lines lacking this phosphotase. Thus, direct evidence showing the biological function and the cellular significance of the enzymatic activity of this signaling protein may be obtained.

Identification of the down-stream targets or substrates of SHP-2 will help to elucidate the biochemical basis of SHP-2 activity in signal transduction. To date, several such molecules have been identified, for instance, SHPS-1[36], PZR[37], and the newly characterized pleckstrin homology domain-containing scaffolding or docking proteins, Gab1[38] and Gab2[39-41]. In fact, Gab2 was originally identified in IL-3 stimulated hematopoietic cells[42], and was shown to be a major SHP-2-binding protein. Subsequently, this strong interaction was further demonstrated to be induced by TPO and EPO stimulation as well as TCR engagement[41]. In addition, the SHP-2/Gab2 complex is also assembled in hematopoietic cells after b1 integrin cross-linking (Qu et al., unpublished data). Our recent studies showed that SHP-1 also associates with Gab2. However, unlike the SHP-2/Gab2 association, SHP-1 and Gab2 are associated in a constitutive manner. Thus both the SHP-2 and SHP-1 phosphatases anchor to the same docking protein Gab2, which may serve as a linker for the functional interaction between these two phosphatases.

\section{Biological functions}

An extensive distribution of SHP-2 phosphatase indicates that it might have a wide range of physiological functions. Recent data from SHP-2 gene knockout mice have clearly suggested this notion. Mice homozygous for a SHP-2 mutation are embryonic lethal. Homozygous mutants die at midgestation with multiple developmental defects in the mesodermal pattering and body organization[43],[44]. A similar requirement for 
SHP-2 in Xenopus development was found to be attributed to its positive role in basic fibroblast growth factor signaling[15]. Chimeric mice generated from homozygous mutant ES cells die at different stages, depending on the contribution from the mutant cells [45]. This strategy has proven to be an alternative approach for further defining the physiological functions that are masked due to the early embryonic lethality of mutant mice. Some interesting phenotypes were observed in chimeric mice containing SHP-2 mutant cells, such as abnormal development of the skeleton and limbs[45],[46]. Another striking observation was that $50 \%$ of the chimeric mice had an open eyelid phenotype, which is a typical phenotype of EGF receptor knockout mice, suggesting that SHP-2 is required for in vivo action of EGF. Further genetic analyses showed that a SHP-2 heterozygous mutation dominantly enhanced the phenotypes of EGF receptor weak allele ( wa-2/ wa-2). Reducing SHP-2 protein levels by half further significantly diminished the EGF signaling on the background of EGF receptor mutation[47]. Additionally, subsequent studies showed that the penetrance and severity of the defective cardiac semilunar valvulogenesis was enhanced in wa-2/wa-2 mice with a heterozygous mutation of SHP-2, indicating a functional requirement for SHP-2 in heart development[48].

In vitro studies have revealed that SHP-2 plays critical roles in regulating a number of cellular activities. ES cells are totipotent embryonic stem cells, that can be induced to differentiate into a variety of cell lineages, including hematopoietic cells, cardiomyocytes, and even neuronocytes. SHP-2 mutation in these stem cells severely decreased erythroid lineage differentiation. Myeloid lineage development was completely blocked[49]. Consistently, in vivo hematopoietic progenitor development of mutant ES cell origin in the bone marrow and fetal liver from the chimeric mice generated from homozygous mutant ES cells was undetectable[45]. Hematopoietic progenitor analyses demonstrated that hematopoietic activity in the yolk sac from homozygous mutant embryos was dramatically decreased, suggesting that the SHP-2 mutation blocks hematopoietic development at the primitive hematopoiesis stage. Moreover, SHP-2 is also required for lymphoid lineage development, as SHP-2 mutation completely blocked the $\mathrm{T}$ and B lymphocyte development in SHP-2-/-/RAG-2-/- chimeric mice. No Thy + T cells and B220 + B cells derived from mutant ES cells were detected, suggesting T and B lymphocyte development was blocked at the Pro-T and Pro-B stages (Qu et al., unpublished data). It seems that these developmental defects occur at very early stages in the differentiation process of ES cells. Indeed, SHP-2 mutation significantly reduced ES cell differentiation potential[30],[50], the differentiation of other lineages, including cardiomyocytes and fibroblasts from mutant ES cells was also decreased.

In contrast with SHP-2 phosphatase, the hematopoietic cell specific-SHP-1 phosphatase negatively regulates hematopoietic cell regulation[51-54]. Functional studies on SHP-1 phosphatase have been considerably aided by analyses of motheaten ( $\mathrm{me} / \mathrm{me}$ ) and viable motheaten $\left(\mathrm{mev}^{v} / \mathrm{mev}^{v}\right)$ mice which have spontaneous mutations in the coding region for the N-terminal SH2 domain and the catalytic domain of SHP-1 respectively 
SHP-2 in Signal transduction

[55]. Homozygous mutants have obvious hematological abnormalities. Both me/me and $\mathrm{mev}^{v} / \mathrm{mev}^{v}$ mice develop systemic autoimmune disease and die after approximately 3 and $9 \mathrm{w}$, respectively[56]. High levels of immunoglobulins-particularly autoantibodies-in peripheral blood and excessive erythropoiesis in spleen suggest a primarily negative regulatory role for this phosphatase in hematopoietic development and function. Consistent with this, it has been found that SHP-1 attenuates signals emanating from receptors for EPO, IL-3, GM-CSF, and M- CSF, and mediates inhibitory signals triggered by immunoglobulin $\gamma$ Fc domains (Fc $\gamma$ RIIB1), NK cell inhibitory receptor, TCR, BCR, CD22, and CD72[52-54], [57-60].

Taken together, these findings suggest that SHP-2 and the homologous SHP-1 phosphatase have opposite functions in regulating hematopoietic cell development, despite sharing high homology. Indeed, recent data from SHP-2/SHP-1 double mutant mice strongly support this notion. Defective primitive hematopoiesis caused by the SHP-2 mutation was partially rescued by an additional SHP-1 mutation (Qu et al., unpublished data). A profound biochemical basis for the distinct functions of these phosphatases remains to be elucidated.

In addition, SHP-2 appears to positively regulate fibroblast cell adhesion and migration[23],[24], [61],[62]. SHP-2 homozygous mutant fibroblast cells have reduced cell spreading and migration. Further biochemical studies have indicated that SHP-2 participates in focal adhesion kinase-mediated integrin signaling. Moreover, SHP-2 is required for in vivo insulin action. Transgenic mice expressing dominant negative SHP2 displayed insulin resistance[25]. Plasma insulin levels in transgenic mice after $4 \mathrm{~h}$ fasting were 3 times greater than none-transgenic controls with comparable blood glucose levels. In the presence of physiological concentration of insulin, the insulin-stimulated glucose uptake in muscle and adipocytes from transgenic mice was impaired.

\section{Perspectives}

It is clear that SHP-2 tyrosine phosphatase plays critical roles in regulating signal transduction, and mediating a variety of cellular biological processes. However, many questions regarding the biochemical basis for its signaling functions still remain unaddressed. Prominent questions are: why does a protein tyrosine phosphatase play a positive role in transducing signal relay from cell surface receptors, and what is the biochemical significance of the phosphatase activity of this enzyme? Importantly, several recent reports have indicated a clinical relevance of SHP-2 phosphatase to some diseases such as neutropenia (Kostmann's syndrome)[63] and diabetes[64],[65], which further emphasizes the importance of studies on the signaling regulation of this PTP. Understanding the profound mechanisms of SHP-2 action will provide novel insights into the regulation of intracellular signaling processes, and may lead to novel molecular therapeutic approaches for certain diseases. 


\section{ACKNOWLEDGEMENTS}

The author thanks Drs. Michael Chase, Kevin Bunting, and Yufang Shi for critical reading of and helpful comments on the manuscript. The author apologizes to many colleagues whose contribution was omitted from this review due to limited space.

\section{REFERENCES}

[1] Hunter T. Signaling--2000 and beyond. Cell 2000; 100:113-27.

[2] Tonks NK and BG Neel. From form to function: signaling by protein tyrosine phosphatases. Cell 1996; 87:365-8.

[3] Denu JM, JA Stuckey, MA Saper, JE Dixon. Form and function in protein dephosphorylation. Cell 1996; 87:361-4.

[4] Feng GS, CC Hui, T Pawson. SH2-containing phosphotyrosine phosphatase as a target of proteintyrosine kinases. Science 1993; 259:1607-11.

[5] Freeman RM, JrJ Plutzky, BG Neel. Identification of a human src homology 2-containing proteintyrosine-phosphatase: a putative homolog of Drosophila corkscrew. Proc Natl Acad Sci USA 1992; 89:11239-43.

[6] Ahmad S, D Banville, Z Zhao, EH Fischer, SH Shen. A widely expressed human protein-tyrosine phosphatase containing src homology 2 domains. Proc Natl Acad Sci USA 1993; 90:2197-201.

[7] Vogel W, R Lammers, J Huang, A Ullrich. Activation of a phosphotyrosine phosphatase by tyrosine phosphorylation. Science 1993; 259:1611-4.

[8] Adachi M, M Sekiya, T Miyachi, K Matsuno, Y Hinoda, K Imai, A Yachi. Molecular cloning of a novel protein-tyrosine phosphatase SH-PTP3 with sequence similarity to the src-homology region 2. FEBS Lett 1992; 314:335-9.

[9] Adachi M, EH Fischer, J Ihle, et al. Mammalian SH2-containing protein tyrosine phosphatases. Cell 1996; 85:15.

[10] Yi T, JL Cleveland, JN Ihle. Identification of novel protein tyrosine phosphatases of hematopoietic cells by polymerase chain reaction amplification. Blood 1991; 78:2222-8.

[11] Koch CA, D Anderson, MF Moran, C Ellis, T Pawson. SH2 and SH3 domains: elements that control interactions of cytoplasmic signaling proteins. Science 1991; 252:668-74.

[12] Hof P, S Pluskey, S Dhe-Paganon, MJ Eck, SE Shoelson. Crystal structure of the tyrosine phosphatase SHP-2. Cell 1998; 92:441-50.

[13] Eck MJ, S Pluskey, T Trub, SC Harrison, SE Shoelson. Spatial constraints on the recognition of phosphoproteins by the tandem SH2 domains of the phosphatase SH-PTP2. Nature 1996; 379:27780.

[14] Tang TL, RM Freeman, JrAM O'Reilly, BG Neel, SY Sokol. The SH2-containing protein-tyrosine phosphatase SH-PTP2 is required upstream of MAP kinase for early Xenopus development. Cell 1995; 80:473-83.

[15] O'Reilly AM and BG Neel. Structural determinants of SHP-2 function and specificity in Xenopus mesoderm induction. Mol Cell Biol 1998; 18:161-77.

[16] Neel BG and NK Tonks. Protein tyrosine phosphatases in signal transduction. Curr Opin Cell Biol 1997; 9:193-204.

[17] Huyer G and DR Alexander. Immune signalling: SHP-2 docks at multiple ports. Curr Biol 1999; 9: R129-32.

[18] Shi ZQ, W Lu, GS Feng. The Shp-2 tyrosine phosphatase has opposite effects in mediating the activation of extracellular signal-regulated and c-Jun NH2-terminal mitogen-activated protein kinases. J Biol Chem 1998; 273:4904-8.

[19] Bennett AM, SF Hausdorff, AM O'Reilly, RM Freeman, BG Neel. Multiple requirements for SHPTP2 


\section{SHP-2 in Signal transduction}

in epidermal growth factor-mediated cell cycle progression. Mol Cell Biol 1996; 16:1189-202.

[20] Hadari YR, H Kouhara, I Lax, J Schlessinger. Binding of Shp2 tyrosine phosphatase to FRS2 is essential for fibroblast growth factor-induced PC12 cell differentiation. Mol Cell Biol 1998; 18:396673.

[21] Milarski KL and AR Saltiel. Expression of catalytically inactive Syp phosphatase in 3T3 cells blocks stimulation of mitogen-activated protein kinase by insulin. J Biol Chem 1994; 269:21239-43.

[22] Yamauchi K, KL Milarski, AR Saltiel, JE Pessin. Protein-tyrosine-phosphatase SHPTP2 is a required positive effector for insulin downstream signaling. Proc Natl Acad Sci USA 1995; 92:664-8.

[23] Inagaki K, T Noguchi, T Matozaki, et al. Roles for the protein tyrosine phosphatase SHP-2 in cytoskeletal organization, cell adhesion and cell migration revealed by overexpression of a dominant negative mutant. Oncogene 2000; 19:75-84.

[24] Manes S, E Mira, C Gomez-Mouton, Z J Zhao, RA Lacalle, AC Martinez. Concerted activity of tyrosine phosphatase SHP-2 and focal adhesion kinase in regulation of cell motility. Mol Cell Biol 1999; 19:3125-35.

[25] Maegawa H, M Hasegawa, S Sugai, et al. Expression of a dominant negative SHP-2 in transgenic mice induces insulin resistance. J Biol Chem 1999; 274:30236-43.

[26] Noguchi T, T Matozaki, K Horita, Y Fujioka, M Kasuga. Role of SH-PTP2, a protein-tyrosine phosphatase with Src homology 2 domains, in insulin-stimulated Ras activation. Mol Cell Biol 1994; 14:6674-82.

[27] Allard JD, HC Chang, R Herbst, H McNeill, MA Simon. The SH2-containing tyrosine phosphatase corkscrew is required during signaling by sevenless, Ras1 and Raf. Development 1996; 122:1137-46.

[28] You M, DH Yu, GS Feng. Shp-2 tyrosine phosphatase functions as a negative regulator of the interferon-stimulated Jak/STAT pathway. Mol Cell Biol 1999; 19:2416-24.

[29] Symes A, N Stahl, SA Reeves, et al. The protein tyrosine phosphatase SHP-2 negatively regulates ciliary neurotrophic factor induction of gene expression. Curr Biol 1997; 7:697-700.

[30] Burdon T, C Stracey, I Chambers, J Nichols, A Smith. Suppression of SHP-2 and ERK signalling promotes self-renewal of mouse embryonic stem cells. Dev Biol 1999; 210:30-43.

[31] Ohtani T, K Ishihara, T Atsumi, et al. Dissection of signaling cascades through gp130 in vivo: reciprocal roles for STAT3- and SHP2-mediated signals in immune responses. Immunity 2000; 12: 95-105.

[32] Nicholson SE, DDe Souza, LJ Fabri, J Corbin, TA Willson, JG Zhang, A Silva, M Asimakis, A Farley, AD Nash, D Metcalf, DJ Hilton, NA Nicola, M Baca. Suppressor of cytokine signaling-3 preferentially binds to the SHP-2-binding site on the shared cytokine receptor subunit gp130. Proc Natl Acad Sci USA 2000; 97:6493-8.

[33] Fuhrer DK, GS Feng, YC Yang. Syp associates with gp130 and Janus kinase 2 in response to interleukin-11 in 3T3-L1 mouse preadipocytes. J Biol Chem 1995; 270:24826-30.

[34] Tauchi T, GS Feng, MS Marshall, et al. The ubiquitously expressed Syp phosphatase interacts with c-kit and Grb2 in hematopoietic cells. J Biol Chem 1994; 269:25206-11.

[35] Tauchi T, GS Feng, R Shen, et al. Involvement of SH2-containing phosphotyrosine phosphatase Syp in erythropoietin receptor signal transduction pathways. J Biol Chem 1995; 270:5631-5.

[36] Fujioka Y, T Matozaki, T Noguchi, et al. A novel membrane glycoprotein, SHPS-1, that binds the SH2-domain- containing protein tyrosine phosphatase SHP-2 in response to mitogens and cell adhesion. Mol Cell Biol 1996; 16:6887-99.

[37] Zhao ZJ and R Zhao. Purification and cloning of PZR, a binding protein and putative physiological substrate of tyrosine phosphatase SHP-2. J Biol Chem 1998; 273:29367-72.

[38] Holgado-Madruga M, DR Emlet, DK Moscatello, AK Godwin, AJ Wong. A Grb2-associated docking protein in EGF- and insulin-receptor signalling. Nature 1996; 379:560-4.

[39] Gu H, JC Pratt, SJ Burakoff, BG Neel. Cloning of p97/Gab2, the major SHP2-binding protein in hematopoietic cells, reveals a novel pathway for cytokine-induced gene activation. Mol Cell 1998; 2: 
729-40.

[40] Zhao C, DH Yu, R Shen, GS Feng. Gab2, a new pleckstrin homology domain-containing adapter protein, acts to uncouple signaling from ERK kinase to Elk-1. J Biol Chem 1999; 274:19649-54.

[41] Nishida K, Y Yoshida, M Itoh, et al. Gab-family adapter proteins act downstream of cytokine and growth factor receptors and T- and B-cell antigen receptors. Blood 1999; 93:1809-16.

[42] Gu H, JD Griffin, BG Neel. Characterization of two SHP- 2- associated binding proteins and potential substrates in hematopoietic cells. J Biol Chem 1997; 272:16421-30.

[43] Saxton TM, M Henkemeyer, S Gasca, et al. Abnormal mesoderm patterning in mouse embryos mutant for the SH2 tyrosine phosphatase Shp-2. Embo J 1997; 16:2352-64.

[44] Arrandale JM, A Gore-Willse, S Rocks, et al. Insulin signaling in mice expressing reduced levels of Syp. J Biol Chem 1996; 271:21353-8.

[45] Qu CK, WM Yu, B Azzarelli, et al. Biased suppression of hematopoiesis and multiple developmental defects in chimeric mice containing Shp-2 mutant cells. Mol Cell Biol 1998; 18:6075-82.

[46] Saxton TM, BG Ciruna, D Holmyard, et al. The SH2 tyrosine phosphatase shp2 is required for mammalian limb development. Nat Genet 2000; 24:420-3.

[47] $\mathrm{Qu}$ CK, WM Yu, B Azzarelli, GS Feng. Genetic evidence that Shp-2 tyrosine phosphatase is a signal enhancer of the epidermal growth factor receptor in mammals. Proc Natl Acad Sci USA 1999; 96: 8528-33.

[48] Chen B, RT Bronson, LD Klaman, et al. Mice mutant for Egfr and Shp2 have defective cardiac semilunar valvulogenesis. Nat Genet 2000; 24:296-9.

[49] Qu CK, ZQ Shi, R Shen, FY Tsai, SH Orkin, GS Feng. A deletion mutation in the SH2-N domain of Shp-2 severely suppresses hematopoietic cell development. Mol Cell Biol 1997; 17:5499-507.

[50] $\mathrm{Qu} \mathrm{CK}$ and GS Feng. Shp-2 has a positive regulatory role in ES cell differentiation and proliferation. Oncogene 1998; 17:433-9.

[51] Kozlowski M, L Larose, F Lee, DM Le, R Rottapel, KA Siminovitch. SHP-1 binds and negatively modulates the c-Kit receptor by interaction with tyrosine 569 in the c-Kit juxtamembrane domain. Mol Cell Biol 1998; 18:2089-99.

[52] Yi T, AL Mui, G Krystal, JN Ihle. Hematopoietic cell phosphatase associates with the interleukin3 (IL-3) receptor beta chain and down-regulates IL-3-induced tyrosine phosphorylation and mitogenesis. Mol Cell Biol 1993; 13:7577-86.

[53] Chen HE, S Chang, T Trub, BG Neel. Regulation of colony-stimulating factor 1 receptor signaling by the SH2 domain-containing tyrosine phosphatase SHPTP1. Mol Cell Biol 1996; 16:3685-97.

[54] Klingmuller U, U Lorenz, LC Cantley, BG Neel, HF Lodish. Specific recruitment of SH-PTP1 to the erythropoietin receptor causes inactivation of JAK2 and termination of proliferative signals. Cell 1995; 80:729-38.

[55] Shultz LD, PA Schweitzer, TV Rajan, et al. Mutations at the murine motheaten locus are within the hematopoietic cell protein -tyrosine phosphatase (Hcph) gene. Cell 1993; 73:1445-54.

[56] Shultz LD. Pleiotropic effects of deleterious alleles at the "motheaten" locus. Curr Top Microbiol Immunol 1988; 137:216-22.

[57] D'Ambrosio D, KL Hippen, SA Minskoff, et al. Recruitment and activation of PTP1C in negative regulation of antigen receptor signaling by Fc gamma RIIB1. Science 1995; 268:293-7.

[58] Doody GM, LB Justement, CC Delibrias, et al. A role in B cell activation for CD22 and the protein tyrosine phosphatase SHP. Science 1995; 269:242-4.

[59] Wu Y, MJ Nadler, LA Brennan, et al. The B-cell transmembrane protein CD72 binds to and is an in vivo substrate of the protein tyrosine phosphatase SHP-1. Curr Biol 1998; 8:1009-17.

[60] Burshtyn DN, AM Scharenberg, N Wagtmann, et al. Recruitment of tyrosine phosphatase HCP by the killer cell inhibitor receptor. Immunity 1996; 4:77-85.

[61] Yu DH, CK Qu, O Henegariu, X Lu, GS Feng. Protein-tyrosine phosphatase Shp-2 regulates cell spreading, igration, and focal adhesion. J Biol Chem 1998; 273:21125-31. 


\section{SHP-2 in Signal transduction}

[62] Hakak Y, YS Hsu, GS Martin. Shp-2 mediates v-Src-induced morphological changes and activation of the anti-apoptotic protein kinase Akt. Oncogene 2000; 19:3164-71.

[63] Tidow N, B Kasper, K Welte. SH2-containing protein tyrosine phosphatases SHP-1 and SHP-2 are dramatically increased at the protein level in neutrophils from patients with severe congenital neutropenia (Kostmann's syndrome). Exp Hematol 1999; 27:1038-45.

[64] Bonini JA, J Colca, C Hofmann. Altered expression of insulin signaling components in streptozotocin-treated rats. Biochem Biophys Res Commun 1995; 212:933-8.

[65] Ahmad F and BJ Goldstein. Alterations in specific protein-tyrosine phosphatases accompany insulin resistance of streptozotocin diabetes. Am J Physiol 1995; 268:E932-40. 